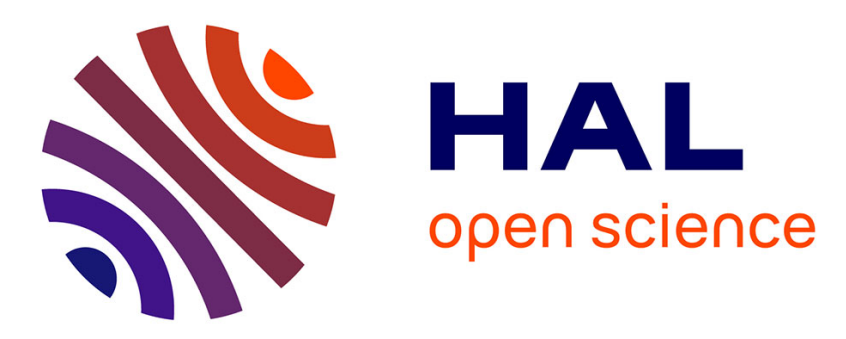

\title{
Wideband multilinear array processing through tensor decomposition
}

\author{
Francesca Raimondi, Pierre Comon, Olivier J.J. Michel
}

\section{To cite this version:}

Francesca Raimondi, Pierre Comon, Olivier J.J. Michel. Wideband multilinear array processing through tensor decomposition. ICASSP 2016 - 41st IEEE International Conference on Acoustics, Speech and Signal Processing, Mar 2016, Shanghai, China. 10.1109/ICASSP.2016.7472218 . hal01206915

\section{HAL Id: hal-01206915 https://hal.science/hal-01206915}

Submitted on 29 Sep 2015

HAL is a multi-disciplinary open access archive for the deposit and dissemination of scientific research documents, whether they are published or not. The documents may come from teaching and research institutions in France or abroad, or from public or private research centers.
L'archive ouverte pluridisciplinaire HAL, est destinée au dépôt et à la diffusion de documents scientifiques de niveau recherche, publiés ou non, émanant des établissements d'enseignement et de recherche français ou étrangers, des laboratoires publics ou privés. 


\title{
WIDEBAND MULTILINEAR ARRAY PROCESSING THROUGH TENSOR DECOMPOSITION
}

\author{
Francesca Raimondi, Pierre Comon, Olivier Michel \\ GIPSA-Lab, CNRS, BP 46, 38402 Saint Martin d'Heres Cedex, France
}

\begin{abstract}
Our goal is to devise a wideband High-Resolution technique that does not require a priori knowledge of DoA rough estimates, and that is able to exploit multiple spatial invariances. Existing tensor array processing techniques are limited to the narrowband case. On the other hand, wideband Esprit has only been proposed with focusing matrices, requiring a priori DoA knowledge. We resort to the decomposition of tensors built on space, space translation and frequency diversities, and demonstrate the good behavior of the algorithm proposed.
\end{abstract}

Index Terms - blind source separation, localization, wideband, DoA estimation, antenna array processing, tensor

\section{INTRODUCTION}

A central problem in array signal processing, in particular for telecommunications, speech, astronomy, seismology, and medical applications, lies in Direction of Arrival (DoA) estimation. An array of multiple sensors placed at different positions in space is conceived to receive source signals from different directions [1], [2]. Some array processing techniques perform an exhaustive search of the angular range, such as beamforming and MUSIC (MUltiple SIgnal Classification) [3], whereas others rely on algebraic solutions, such as rootMUSIC [4] and ESPRIT [5].

Signal-subspace processing embodied by MUSIC and ESPRIT exploits the algebraic property of the spatial covariance matrix that the eigenvectors corresponding to the largest eigenvalues span the same subspace as the source direction vectors. The rotational invariance principle of ESPRIT was extended to more than one displacement through tensor decomposition of narrowband waves [6]. This deterministic approach provides the localization of more sources than sensors in each subarray, without requiring signal stationarity (which is the underlying assumption of the traditional statistical methods). Tensor decompositions can hence potentially handle shorter data records, since they do not resort to the estimation of statistical quantities from available samples.

For wideband signals, signal-subspaces are different at different frequencies, and the afore mentioned narrowband methods need to be adapted to deal with this diversity. The

\footnotetext{
This work has been supported by ERC AdG-2013-320594 grant "DECODA".
}

concept of signal-subspace, originally derived for $R$ narrowband and stationary signals, can be generalized to the wideband case, and need a prior estimate of DoA to form focusing matrices $[7,8,9]$. Another approach consists in building virtual arrays via spatial interpolation, so as to align steering vectors for all frequencies. The advantage of the latter is that no prior estimate is required. However, this kind of approach has only been proposed for MUSIC $[10,11]$.

Our goal is to develop a high resolution deterministic method for wideband source extraction and localization, that could take advantage of particular structures of antenna arrays, without needing prior DoA estimates nor requiring exhaustive search. The contribution of this paper is to extend narrowband tensor decomposition for DoA estimation to the wideband processing of plane waves in the far field, through spatial interpolation. Moreover, the proposed method provides joint source estimates without further processing.

Notation We shall assume throughout the following notations: matrices, column vectors and scalars are denoted respectively in bold uppercase, e.g. $\boldsymbol{A}$, bold lowercase, e.g. $\boldsymbol{v}$, and plain lowercase; in particular array entries are written e.g. $v_{j}$ or $A_{i j}$. Transposition, complex conjugation and Hermitian transposition are denoted by $\left({ }^{\top}\right),\left({ }^{*}\right)$ and $\left({ }^{\mathrm{H}}\right)$, respectively. Arrays with more than two indices are referred to as tensors, with some abuse of terminology [12], and are denoted in bold calligraphic, as $\mathcal{T}$. The outer (tensor) product between two vectors is denoted by $\boldsymbol{u} \otimes \boldsymbol{v}$. For the sake of conciseness, $\boldsymbol{a}_{i}$ will represent the $i$-th column of matrix $\boldsymbol{A}$. Finally, $\|\cdot\|_{F}$ refers to the Frobenius norm.

\section{MULTIPLE-SOURCE MULTIPLE-SENSOR}

Differential travel time from the reference sensor $(l=1)$ to the $l$-th element of the array is given by $\tau_{l}=\left(\boldsymbol{b}_{l}^{T} \boldsymbol{u}\right) / c$ where $\boldsymbol{b}_{l}$ is the position of the $l$-th sensor, $\boldsymbol{u}$ is the unit steering vector and $c$ the constant propagation speed. For an uniform linear array (ULA), it becomes

$$
\tau_{l}(\theta)=\frac{d}{c}(l-1) \sin \theta
$$

where $d$ denotes the inter-sensor distance and $\theta$ the impinging direction. In the presence of noise, the model for a single wideband source recorded by the $l$-th sensor is expressed by

$$
y_{l}(t)=s\left(t-\tau_{l}(\theta)\right)+n_{l}(t), 1 \leq l \leq L
$$


where $\tau_{l}(\theta)$ is the receiving delay at the $l$-th sensor in (1). After applying the Fourier Transform to the data $\boldsymbol{y}(t)$, we obtain

$$
\boldsymbol{y}(f)=\boldsymbol{a}(f) s(f)+\boldsymbol{n}(f), f=1, \ldots, K
$$

where vector $\boldsymbol{a}(f)$ represents the array response to the propagation of a narrow band plane wave arriving from direction $\boldsymbol{u}$ at frequency $f$.

In the presence of $R$ narrowband sources around the same frequency $f$, the resulting signal is the superimposition:

$$
\boldsymbol{y}(f)=\sum_{r=1}^{R} \boldsymbol{a}_{r}(f) s_{r}(f)+\boldsymbol{n}(f)=\boldsymbol{A}(f) \boldsymbol{S}(f)+\boldsymbol{n}(f)
$$

where $\boldsymbol{A}(f)=\left[\boldsymbol{a}_{1}(f), \cdots, \boldsymbol{a}_{R}(f)\right]$ refers to the steering matrix and $\boldsymbol{S}(f)=\left[S_{1}(f), \ldots, S_{r}(f)\right]^{\top}$ to the source amplitude Fourier coefficients. The steering vector for the $r$-th source is given by

$\boldsymbol{a}_{r}(f)=\left[1, \exp \left\{\frac{i 2 \pi f}{c} \boldsymbol{b}_{2}^{\top} \boldsymbol{u}_{r}\right\}, \cdots, \exp \left\{\frac{i 2 \pi f}{c} \boldsymbol{b}_{L}^{\top} \boldsymbol{u}_{r}\right\}\right]^{\top}$

where $\boldsymbol{u}_{r}$ refers to the direction of the $r$-th source; it has a Vandermonde structure when the array is an ULA, according to (1). For convenience, data are arranged in a $L \times K$ matrix $\boldsymbol{Y}=[\boldsymbol{y}(1), \ldots, \boldsymbol{y}(K)]$, where $K$ denotes the number of frequency bins.

\section{WIDEBAND DOA ESTIMATION}

Wideband beamforming (BF) builds a specific spatial response of the array (i.e. beam pointing to the desired source, and hopefully nulls towards other interferences) in order to estimate the signature of the sources impinging on the array in the presence of noise. An exhaustive search involves the pre-steering of the array in a given look direction $\theta \in$ $[-\pi / 2,+\pi / 2]$, by applying a sensor dependent time delay $T_{l}(\theta)=T_{0}+\tau_{l}(\theta)$

$$
y_{l}^{p s}(t, \theta)=y\left(t+\tau_{l}\left(\theta_{r}\right)-T_{l}(\theta)\right) \quad l=0, \ldots, L-1
$$

where $T_{0}$ is a bulk delay such that $T_{l}(\theta)>0, \forall l$ [13]. A time interpolation is then necessary for a correct implementation of presteering delays in order to extrapolate signal values at given time delays between two original samples. The effect of presteering in a source direction $\theta_{r}$ is a spatial alignment of all the waveforms on all sensors, corresponding to a local maximum energy output when $\theta=\theta_{r}: y_{l}^{p s}\left(t, \theta_{r}\right) \approx s_{r}(t-$ $\left.T_{0}\right), \forall l$. The output of narrowband BF is a linear combination of spatial samples:

$$
y^{B F}(t, \theta)=\sum_{l=0}^{L-1} y_{l}^{p s}(t, \theta) w_{l}
$$

However, when the signal bandwidth increases, its performance degrades. One of the first wideband solutions was provided for this purpose in [1] and involves a Tapped Delay Line
(TDL) scheme (or FIR filters as for the discrete case). When the number of sources increases, the performance of time domain beamforming rapidly degrades.

High Resolution (HR) methods such as MUSIC and ESPRIT have been originally designed in narrow band. Two wideband extensions can be considered, which jointly process the information in all frequency bands:

a) Incoherent signal-subspace processing: the wide frequency band can be divided into non-overlapping narrowbands, and narrowband signal subspace methods are applied in each narrowband separately. All the results from individual narrowband processing are then combined through an average operation [7].

b) Coherent Signal-Subspace (CSS) [8, 14, 9] relies on an approximately coherent combination of the spatial signal subspaces at different frequencies through the MUSIC algorithm. Through the same CSS process, ESPRIT was extended to the wideband case by $[15,9]$. The CCS proposed by [8] performs an alignment of each steering vector associated with the narrowband signal component propagating at frequency $f$, to a reference frequency $f_{0}$ through a linear transform $\boldsymbol{T}\left(f, f_{0}\right)$ satisfying:

$$
\boldsymbol{T}\left(f, f_{0}\right) \boldsymbol{A}(f)=\boldsymbol{A}\left(f_{0}\right)
$$

Therefore, all the components of a wideband signal, propagating at different frequencies, are related to the same coherent signal-subspace (CSS) at reference frequency $f_{0}=c / \lambda_{0}$. This method has the disadvantage of requiring a prior rough estimate of the DoAs, $\theta_{r}$, in order to calculate linear transformations $\boldsymbol{T}\left(f, f_{0}\right)$.

We will show in Section 4 that focusing received signals onto a CSS can be substituted by a spatial interpolation between sensors, that does not require any prior rough estimation of DoAs. From this perspective, the time interpolation underlying wideband beamforming converts to a spatial interpolation for frequency domain wideband methods.

Whereas statistical high resolution methods resort to the estimation of the covariance matrix, thus requiring long observation snapshots, tensor methods are deterministic. An illustration may be found in [16], where the diversity of propagation speed was exploited to perform an alignment onto the same common subspace.

\section{SPATIAL INTERPOLATION}

As pointed out in [10], the CSS approach through focusing matrices can be replaced by a spatial interpolation in wideband MUSIC for an ULA. In fact, the CSS can be built by introducing a modified sampling interval $d(f) \neq d_{0}$, so that the product $f d(f)$ is kept constant, without any prior assumption on the position of the sources:

$$
d(f)=d_{0} \frac{f_{0}}{f}=d_{0} \frac{\lambda}{\lambda_{0}}
$$

where $d_{0}$ is the nominal inter-sensor distance of the array and $\lambda_{0}$ is the reference wavelength of the CSS. The same idea 
was exploited in [11], where the approach is also an alignment of broadband sources in the spatial frequency domain, by treating the output of a discrete array as the result of spatial sampling of a continuous array.

The Fourier Transform of the wideband field incident on the ULA at position $x$ in space at frequency $f$ is then

$$
y(x, f)=\sum_{r=1}^{R} s_{r}(f) \exp \left\{i \frac{2 \pi}{c} f x \sin \theta_{r}\right\}+n(x, f),
$$

which yields a discretized expression for $x=(l-1) d_{0}$.

The result of the resampling of the sensor output with sensor spacing $d(f)$ is an alignment of the steering matrix, that becomes constant for all the considered frequencies within the common bandwidth of the sources. The adjusted sensor output

$\tilde{y}(l, f)=\sum_{r=1}^{R} \tilde{s}_{r}(f) \exp \left\{i \frac{2 \pi}{c} f_{0} d_{0}(l-1) \sin \theta_{r}\right\}+\tilde{n}(l, f)$

leads to a constant focused steering matrix $\tilde{\boldsymbol{A}}(f)=\boldsymbol{A}\left(f_{0}\right)$ in the system (2)

$$
\tilde{\boldsymbol{y}}\left(f_{k}\right)=\boldsymbol{A}\left(f_{0}\right) \tilde{\boldsymbol{s}}\left(f_{k}\right)+\tilde{\boldsymbol{n}}\left(f_{k}\right), 1 \leq k \leq K
$$

where $K$ is the number of frequency bins of the Fourier Transform. An average $L \times L$ covariance matrix focused at frequency $f_{0}$ may be calculated in $[10,11]$ as

$$
\tilde{\boldsymbol{R}}\left(f_{0}\right)=\frac{1}{K} \sum_{k=1}^{K} \tilde{\boldsymbol{y}}\left(f_{k}\right) \tilde{\boldsymbol{y}}\left(f_{k}\right)^{\mathrm{H}} .
$$

In addition to frequency smoothing, a preliminary spatial smoothing can be applied $[17,18]$ :

$$
\tilde{\boldsymbol{R}}^{(s)}\left(f_{0}\right)=\frac{1}{K} \sum_{k=1}^{K} \frac{1}{M} \sum_{m=1}^{M} \tilde{\boldsymbol{y}}_{m}\left(f_{k}\right) \tilde{\boldsymbol{y}}_{m}\left(f_{k}\right)^{\mathrm{H}}
$$

where index $m$ refers to the $m$-th subarray within the ULA. Note that the values that would occur at virtual positions $d\left(f_{k}\right)$ satisfying (3) are estimated, provided that the Nyquist criterion is satisfied: in order to avoid spatial aliasing, the spatial period must always respect the condition $d\left(f_{k}\right) \leq$ $\frac{1}{2} \frac{c}{f_{k}}, \forall k$, which is equivalent to $d_{0} \leq \lambda_{0} / 2$ from (3).

\section{WIDEBAND TENSOR PROCESSING THROUGH SPATIAL INTERPOLATION}

The tensor model expresses a structured phenomenon through finite sums and products of simpler constituents [19] (parsimony and separability). The physical measurements are stored in a multidimensional array and decomposed into a sum of rank one or decomposable terms [12,6]. A decomposable three-way tensor can be defined by a vector triplet

$$
D_{k l m}=s_{k} a_{l} b_{m} \text { or equivalently } \mathcal{D}=\boldsymbol{s} \otimes \boldsymbol{a} \otimes \boldsymbol{b}
$$

Any order-3 tensor admits a decomposition into a sum of decomposable tensors $\mathcal{T}=\sum_{r=1}^{R} \varsigma_{r} \mathcal{D}(r)$. In array processing, $R$ refers to the number of sources, correlated or not [19].

The minimal value of $R$ such that this decomposition holds is called the tensor rank: if $R$ is not too large, the corresponding decomposition is unique and is referred to as Canonical Polyadic (CP) [12]. Once bases in every linear space are fixed, tensor $\mathcal{T}$ is represented by a $K \times L \times M$ three-way array, which consequently decomposes as

$$
\mathcal{T}_{k l m}=\sum_{r=1}^{R} \varsigma_{r} S_{k r} A_{l r} B_{m r}
$$

This is equivalent to the general $R$-term trilinear model

$$
\mathcal{T}=\sum_{r=1}^{R} \varsigma_{r} \boldsymbol{s}_{r} \otimes \boldsymbol{a}_{r} \otimes \boldsymbol{b}_{r}
$$

where each term $\boldsymbol{s}_{r} \otimes \boldsymbol{a}_{r} \otimes \boldsymbol{b}_{r}$ has rank 1.

The core of this paper lies in the combination of several elements: introduction of the frequency diversity into the separable model, spatial interpolation for each narrowband so as to align (focus) their respective contributions, and then a tensor joint processing leading to DoA estimation.

In addition to space and frequency diversities, we exploit the space shift diversity described in $[6,20]$ : the entire array structure is composed of a reference subarray repeated in space through $M$ translations, $\boldsymbol{\delta}_{m}$, extending the same rotational invariance as ESPRIT [5] to more than one displacement. However, unlike the deterministic model in [6], we herein apply tensor analysis to Fourier transformed data, and work in the frequency domain. This leads to the tensor model:

$\mathcal{T}_{k l m}=\sum_{r=1}^{R} s_{r}\left(f_{k}\right) \exp \left\{i \frac{2 \pi f_{k}}{c} \boldsymbol{b}_{l}^{T} \boldsymbol{u}_{r}\right\} \exp \left\{i \frac{2 \pi f_{k}}{c} \boldsymbol{\delta}_{m}^{T} \boldsymbol{u}_{r}\right\}$

If the array is an $L$-sensor ULA, it can be considered as the combination of $M$ subarrays with $L-M+1$ sensors each. After spatial interpolation, we have:

1. $\varsigma_{r} S_{k r}=\tilde{s}_{r}\left(f_{k}\right)$ is the $k$-th FT component of the $r$ th interpolated signal. Since the number of frequency components is $K, \boldsymbol{S} \in \mathbb{C}^{K \times R}$.

2. $A_{l r}=\exp \left\{i \frac{2 \pi f_{k} d\left(f_{k}\right)}{c}(l-1) \sin \left(\theta_{r}\right)\right\}$ is a scalar entry of the steering matrix for $L-M+1$ sensors ( $\boldsymbol{A} \in$ $\left.\mathbb{C}^{L \times R}\right)$.

3. $B_{m r}=\exp \left\{i \frac{2 \pi f_{k} d\left(f_{k}\right)}{c}(m-1) \sin \left(\theta_{r}\right)\right\}$ refers to the $m$-th space shift $\left(\boldsymbol{B} \in \mathbb{C}^{M \times R}\right)$.

As pointed out in Section 4, the distance between two successive sensors within each subarray must be less than or equal to $\lambda_{0} / 2$ (condition to avoid spatial aliasing).

The matrix parameters of the trilinear problem $\boldsymbol{S}, \boldsymbol{A}$, and $\boldsymbol{B}$ are extracted through the tensor decomposition technique detailed in [21]. DoAs can then be estimated in a second stage through a Least Squares (LS) or Total Least Squares (TLS) solution [20]. 


\section{COMPUTER RESULTS}

We simulate $R=2$ white Gaussian unit-variance correlated sources (correlation coefficient of 0.97), at the sampling frequency $\mathrm{f}_{s}=1 \mathrm{kHz}$, during an observation time of $64 \mathrm{~ms}$ and propagating at $c=1800 \mathrm{~m} / \mathrm{s}$ (propagation speed of S-waves in ice). The number of frequency bins is $K=17$ (from $f_{\min }=0.2 \mathrm{f}_{s}$ to $f_{\max }=0.5 \mathrm{f}_{s}$ ), and the number of space shifts is $M \in\{2,3\}$. Spatial Nyquist conditions is ensured by $d_{0} \leq \lambda_{\max } / 2$ where $\lambda_{\max }=c / f_{\min }$. Interpolation is performed using the Shannon-Whittaker formula [22] in the band $\left[-f_{\max }, f_{\max }\right]$. The acquisition system is given by an ULA of $L$ sensors, where the following space invariances are considered: 2 ULA subarrays of $L-1$ sensors (labelled "CP2"), or 3 ULA subarrays of $L-2$ sensors (labelled "CP3"). The label "SS" specifies that spatial smoothing (4) has been performed with $M=3$.

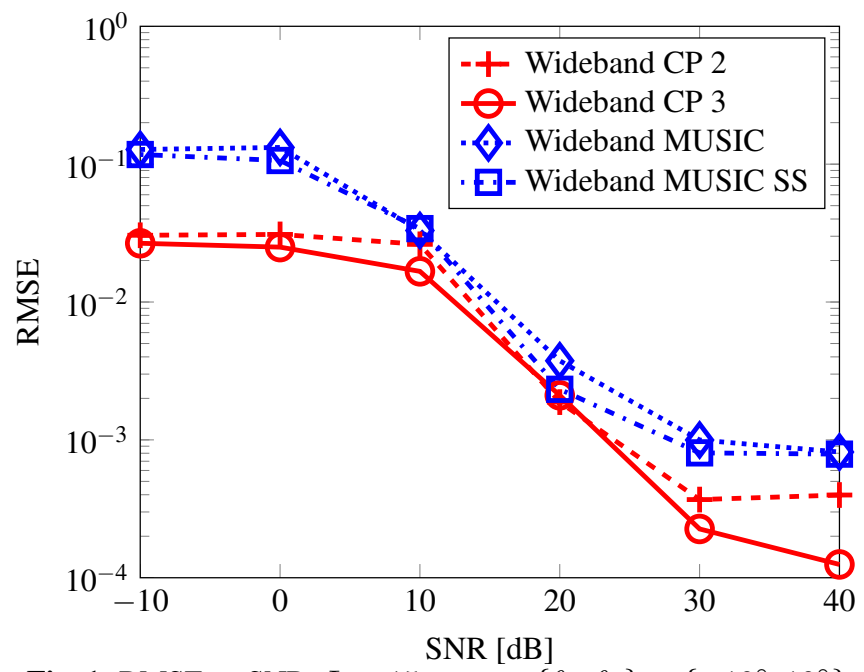

Fig. 1: RMSE vs SNR, $L=15$ sensors, $\left\{\theta_{1}, \theta_{2}\right\}=\left\{-10^{\circ}, 10^{\circ}\right\}$

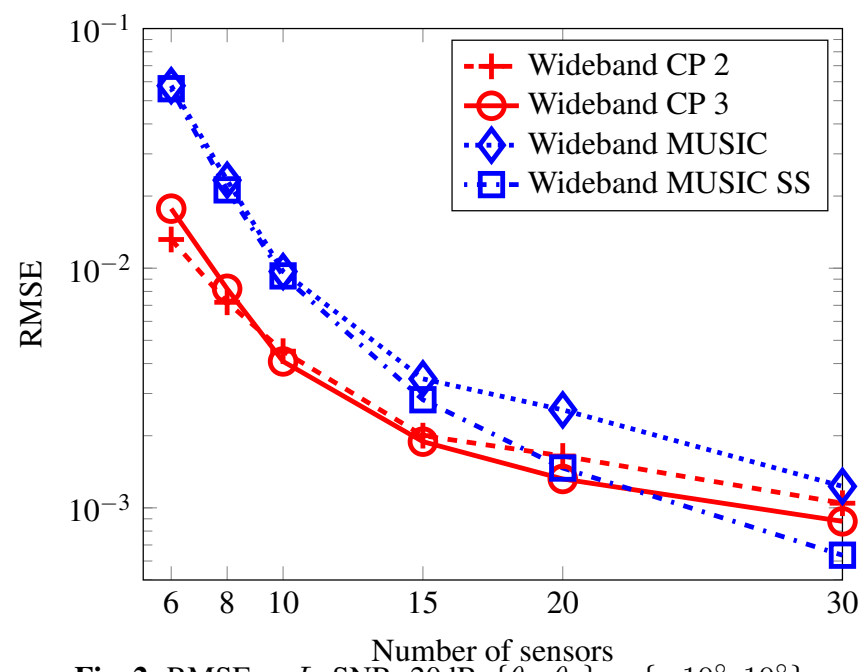

Fig. 2: $\operatorname{RMSE}$ vs $L, \mathrm{SNR}=20 \mathrm{~dB},\left\{\theta_{1}, \theta_{2}\right\}=\left\{-10^{\circ}, 10^{\circ}\right\}$

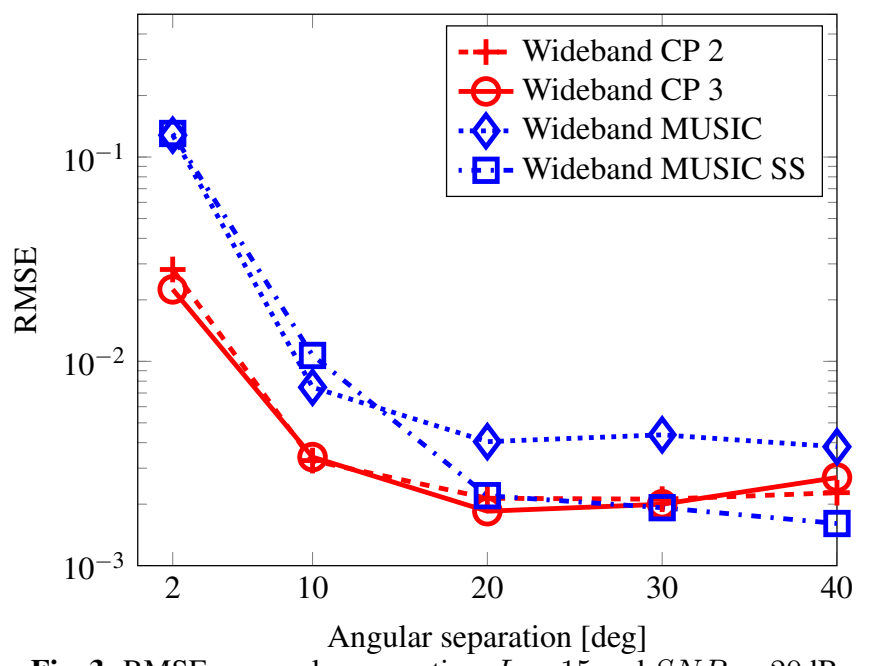

Fig. 3: RMSE vs angular separation, $L=15$ and $S N R=20 \mathrm{~dB}$

The reference frequency is chosen to be $f_{0}=f_{\min }=$ $0.2 \mathrm{f}_{s}$, such that $d_{k} \leq d_{0}, \forall k$. Estimation efficiency is evaluated in comparison to wideband MUSIC and the performance criterion is the relative average Root Mean Square Error (RMSE) of the DoA:

$$
\operatorname{RMSE}(\boldsymbol{\theta})=\frac{1}{\theta_{\max }} \sqrt{\frac{1}{N R} \sum_{n=1}^{N} \sum_{r=1}^{R}\left(\hat{\theta}_{r n}-\theta_{r}\right)^{2}},
$$

where $\hat{\theta}_{r n}$ is the estimated DoA of source $r$ in the $n$-th MonteCarlo trial, $N=200$ being the number of trials, and $\theta_{\max }=$ $\pi=180^{\circ}$. The SNR definition below is assumed:

$$
S N R=10 \log _{10} \frac{\|\mathcal{T}\|_{F}^{2}}{K L M \sigma_{n}^{2}} .
$$

The tensor decomposition with $M=3$ spatial translations yields better results when the number of sensor or the SNR increase, as can be seen in Figures 1 and 2. A slight saturation or deterioration of the performance of the DoA algorithms take place when the two sources move away from the broadside $\left(\theta_{r} \approx 0^{\circ}\right)$, as demonstrated in Fig. 3. Tensor-based DoA estimation outperforms MUSIC, especially for closely spaced sources and for a small number of sensors.

\section{CONCLUSIONS}

We implemented the spatial interpolation technique in wideband tensor-based algorithms. Space and frequency smoothing have been realized in a deterministic framework.

However, we studied so far only scenarios in which subarrays are part of a unique ULA. In this particular configuration, spatial interpolation is easier to handle. Current works include investigations of more general array structures, where subarrays can be disjoint, and in-depth analysis of the influence of source correlation and sensor spacing. 


\section{REFERENCES}

[1] W. Liu and S. Weiss, Wideband beamforming: concepts and techniques, vol. 17, John Wiley \& Sons, 2010.

[2] H. Krim and M. Viberg, "Two decades of array signal processing research: the parametric approach," IEEE Sig. Proc. Magazine, vol. 13, no. 4, pp. 67-94, 1996.

[3] R. O. Schmidt, "Multiple emitter location and signal parameter estimation," IEEE Trans. Ant. Prop., vol. 34, no. 3, pp. 276-280, Mar. 1986.

[4] A. Barabell, "Improving the resolution performance of eigenstructure-based direction-finding algorithms," in ICASSP, Boston, 1983, vol. 8, pp. 336-339.

[5] R. Roy and T. Kailath, "ESPRIT-estimation of signal parameters via rotational invariance techniques," IEEE Trans. Acoust. Speech Sig. Proc., vol. 37, no. 7, pp. 984995, 1989.

[6] N. D. Sidiropoulos, R. Bro, and G. B. Giannakis, "Parallel factor analysis in sensor array processing," IEEE Trans. Sig. Proc., vol. 48, no. 8, pp. 2377-2388, Aug. 2000 .

[7] B. D. Van Veen and K. M. Buckley, "Beamforming: A versatile approach to spatial filtering," IEEE Acoust. Speech Sig. Proc. Magazine, vol. 5, no. 2, pp. 4-24, 1988.

[8] H. Wang and M. Kaveh, "Coherent signal-subspace processing for the detection and estimation of angles of arrival of multiple wide-band sources," IEEE Trans. Acoust. Speech Sig. Proc., vol. 33, no. 4, pp. 823-831, 1985.

[9] B. Ottersten and T. Kailath, "Direction-of-arrival estimation for wide-band signals using the esprit algorithm," IEEE Trans., vol. 38, no. 2, pp. 317-327, 1990.

[10] H. Clergeot and O. Michel, "New simple implementation of the coherent signal subspace method for wide band direction of arrival estimation," in ICASSP. IEEE, 1989, pp. 2764-2767.

[11] J. Krolik and D. Swingler, "Focused wide-band array processing by spatial resampling," IEEE Trans. Acoust. Speech Sig. Proc., vol. 38, no. 2, pp. 356-360, 1990.

[12] P. Comon, "Tensors: a brief introduction," in IEEE Sig. Proc. Magazine, 2014, vol. 31, pp. 44-53.

[13] L. C. Godara, "Application of antenna arrays to mobile communications. ii. beam-forming and directionof-arrival considerations," Proceedings of the IEEE, vol. 85, no. 8, pp. 1195-1245, 1997.
[14] M.A. Doran, E. Doron, and A. J. Weiss, "Coherent wide-band processing for arbitrary array geometry," IEEE Trans. Sig. Proc., vol. 41, no. 1, pp. 414, 1993.

[15] H. Hung and M. Kaveh, "Coherent wide-band esprit method for directions-of-arrival estimation of multiple wide-band sources," IEEE Trans. Acoust. Speech Sig. Proc., vol. 38, pp. 354-356, 1990.

[16] F. Raimondi, P. Comon, O. Michel, S. Sahnoun, and A. Helmstetter, "Tensor decomposition exploiting diversity of propagation velocities: Application to localization of icequake events," Signal Processing, vol. 118, pp. $75-88,2016$.

[17] T.-J. Shan, M. Wax, and T. Kailath, "On spatial smoothing for direction-of-arrival estimation of coherent signals," IEEE Trans. Acoust. Speech Sig. Proc., vol. 33, no. 4, pp. 806-811, 1985.

[18] A. J. Weiss and B. Friedlander, "Performance analysis of spatial smoothing with interpolated arrays," IEEE Trans. Sig. Proc., vol. 41, no. 5, pp. 1881-1892, 1993.

[19] L.-H. Lim and P. Comon, "Blind multilinear identification," IEEE Trans. Inf. Theory, vol. 60, no. 2, pp. 1260-1280, Feb. 2014, open access. hal-00763275.

[20] S. Sahnoun and P. Comon, "Joint source estimation and localization," IEEE Trans. Sig. Proc., vol. 63, no. 10, pp. 2485-2495, 2015.

[21] S. E. Leurgans, R. T. Ross, and R. B. Abel, "A decomposition for three-way arrays," SIAM J. Matrix Ana. Appl., vol. 14, no. 4, pp. 1064-1083, 1993.

[22] C. E. Shannon, "Communication in the presence of noise," Proceedings of the IRE, vol. 37, no. 1, pp. 10-21, 1949. 